\title{
Effects of Microstructure Characteristics of Cortical Bone on its Microcrack Propagation
}

\author{
Yu-xi Liu', Ai-hua Li², Yan-hua $\mathrm{Li}^{3 *}$ \\ ${ }^{1}$ College of Mechanical Engineering, Chongqing Vocational Institute of Engineering, Chongqing 402260, China \\ ${ }^{2}$ Key Laboratory for Biorheological Science and Technology of Ministry of Education, Chongqing University Cancer Hospital, \\ Chongqing 400030, China \\ ${ }^{3}$ Ministry of Education Key Laboratory of Child Development and Disorders, Chongqing Key Laboratory of Translational Medical \\ Research in Cognitive Development and Learning and Memory Disorders, China International Science and Technology Cooperation \\ base of Child development and Critical Disorders, Children's Hospital of Chongqing Medical University, Chongqing 400014, China
}

\begin{abstract}
Scanning electron microscope (SEM) was used to observe and analyze the microstructure of the cross section of cortical bone. The observation results illustrated that the cortical bone is composed of cylindrical osteons and interstitial bone between osteons, and the osteon are unevenly distributed. Based on the microstructure characteristics of cortical bone, three types of cortical bone mesoscopic analysis models were established. Then, the extended finite element method (X-FEM) was used to simulate the microcrack propagation process in bone. The simulate results show that the crack initiation strain of the two-phase model is $19.1 \%$ larger than that of the single-phase model, and the three-phase model is $57.8 \%$ larger than that of the two-phase model, which demonstrated that the osteons and cement line can significantly enhance the crack initiation strain of bone. In addition, under the same boundary conditions, the model with cement line can effectively change the propagation path of microcrack and prevent the propagation of crack. Therefore, the cement lines in cortical bone can effectively increase the fracture resistance of bone and enhance the fracture toughness of cortical bone.
\end{abstract}

\section{Introduction}

Bone is a hierarchical biomaterial that exhibits different fracture mechanisms at each scale [1]. The strength and fracture toughness of bone effected by its microstructure characteristics and microstructural parameters. For understanding the fracture behavior of the bone, it is necessary to evaluate the impact of the microstructural characteristics of the bone on the fracture process.

Cortical bone is located outside of bone and is the main load-bearing material in the bone, which is composed of osteon and interstitial bone on a meso-scale [2]. Due to the important role of cortical bone in bone bearing, there are many research reports on the mechanical behavior of cortical bone [3-5]. Libonati et al. [6] measured the fracture toughness of cortical bone under different loading modes and analyzed the experimental results. Demirtas et al. [7] evaluated the effects of varying the number of unique elastic and fracture properties for osteons, interstitial bone, and cement lines on fracture resistance of cortical bone using finite element modeling. Najafi et al. [8] established a cortical bone mechanical model containing a single osteon and multiple radial microcracks based on linear elastic fracture mechanics, analyzed the effect of the osteon system on the radial microcrack propagation process. Liu et al. [9] used compression experiments and meso-mechanical analysis to investigate the relationships between the microstructural characteristics of bovine femur cortical bone and its mechanical behavior.

In view of the shortcomings of the finite element method in dealing with discontinuous interfaces and in order to study structural fractures more effectively, an extended finite element method [10-12] was proposed in some literatures to analyze the fracture process of materials. Budyn et al. [11] established a multi-scale model of cortical bone based on the extended finite element method, and used it to simulate multiple crack growth in human cortical bone under tension. The results show that the microstructural characteristics of bone effect its failure and fracture. Abdel-Wahab et al. [12] established a crack propagation model of cortical bone microstructure based on linear elastic mechanics and extended finite element theory, and the relationships between the heterogeneity of cortical bone microstructure and microcrack propagation path were investigated. Li et al. [13] established a finite element fracture model to simulate the failure process of a cortical bone specimen in a three-point bending experiment, the analysis results agree well with the experimental data.

At present, in the existing literatures, the research on the mechanical behavior of cortical bone crack propagation mainly uses linear elastic fracture mechanics, and the established cortical bone models do not include Haver's canals and cement lines. Thus, this cannot reflect

\footnotetext{
* Corresponding author: huahua062883@sohu.com
} 
the influence of the true structural characteristics of cortical bone on microcracks propagation. In view of the insufficient research in the existing literatures, this paper investigates the effect of osteon and cement lines on the meso-scale of cortical bone on crack propagation. A single-phase model, a two-phase model of osteon-bone interstitial and a three-phase model of osteon-bone interstitial-cement line were established. The Abaqus XFEM was used to calculate the stress distribution and crack initiation strain in the three models. The crack propagation processes in the two models and the threephase model were simulated, and the effects of osteon and cement lines on the microcrack propagation path were compared and analyzed.

\section{Methods and Material}

\subsection{Microstructure of cortical bone}

Six samples were taken along the axial direction of compact bone of bovine femur (the angle between the length direction of the sample and the axis is $0^{\circ}$ ), then, an external force is applied to bend and break in a direction perpendicular to the axis based on three-point bend test. Then, scanning electron microscope (TESCAN VEGA 3 LMH, SEM) was used to observe and analyze the fracture surface of the samples. SEM observation of the fracture surface of the cortical bone unveils that cortical bone is composed of a cylindrical osteon and the interstitial bone between the osteon (Fig. 1). The osteons are unevenly distributed in the interstitial bone, and osteons and interstitial bone constitute the primary structure of cortical bone. Further observation of the osteon showed that the osteon was composed of multilayer bone lamella surrounding the Haversian canal (Fig. 1b). There are clear boundaries between the osteons and the interstitial bone outside them, these boundaries are called cement lines [14], which constitute the secondary structure of cortical bone.

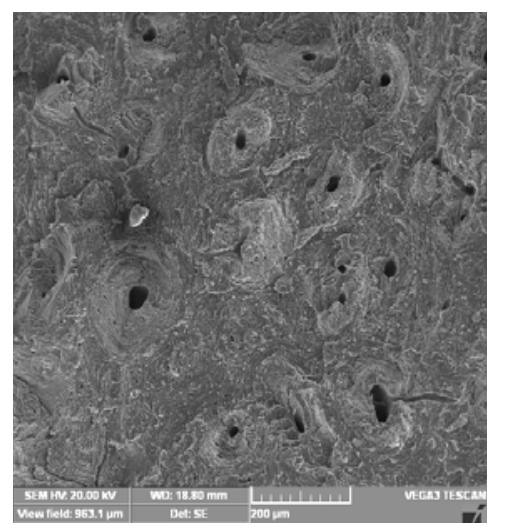

(b)

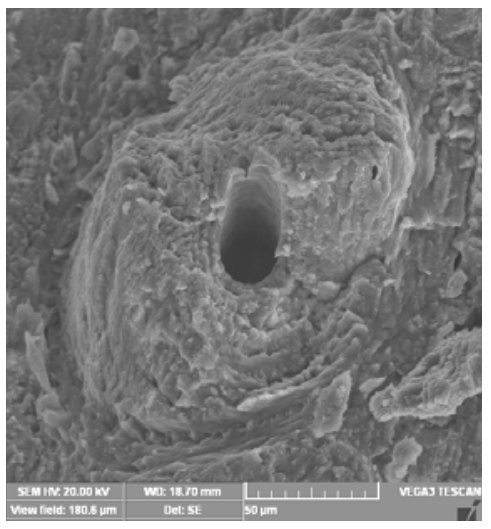

Fig. 1. Microstructure of cortical bone. (a) The fracture surface of cortical bone, (b) microstructure of osteon

\subsection{Analysis model and parameters}

In order to compare and analyze the influence of cement line and osteon on the crack propagation path, three different analysis models were established: single-phase model, two-phase model of osteon-bone interstitial model, and three-phase model of osteon-bone interstitialcement line. A microcrack was added near the osteon, and then take a quarter of the model to establish a mesoscale analysis model. The Abaqus X-FEM is applied to simulate the crack propagation process in cortical bone, and the effects of cement lines and osteon on crack propagation paths in cortical bone were investigated.

The two-phase meso-analysis model and the threephase meso-analysis model are shown in Fig. 2 and Fig. 3. The appearance and structural parameters of the three analysis models are shown in Fig. 2 and Fig. 3. The model boundary size is $125 \mu \mathrm{m} \times 125 \mu \mathrm{m}$; the radius of the osteon is $80 \mu \mathrm{m}$ [15] and the radius of the Haversian canal is $20 \mu \mathrm{m}$. The thickness of cement line is $5 \mu \mathrm{m}$. An initial crack with a length of $20 \mu \mathrm{m}$ was preset at the same position of the three models. Because the model is symmetrical about $x$-axis and $y$-axis, its boundary conditions are: along the left edge of the model, the displacement in the $x$ direction and $y$ direction are restricted, and the degree of freedom of rotation around the $y$-axis and the $z$-axis are also constrained (Fig. 2b and Fig. 3b). A uniform tensile stress of $35 \mathrm{MPa}$ on the right side of the model was applied, and set a point at the midpoint on the right side of the model as a reference point for the output of the calculation results. Then, the three models were analyzed separately, and the effects of the cement line on the crack propagation path in different models were compared.

When the X-FEM was used to analyze the process of crack propagation, it is necessary to set crack damage initiation and damage evolution criteria to achieve crack propagation. The study found that the fracture failure process of cortical bone is based on the control of strain parameters [16]. Therefore, the maximum principal strain failure criterion are used in the above models as the crack initiation criteria. The crack initiation strain of the bone interstitial, osteon and cement line is set as $0.4 \%$ [12], and the damage evolution process of the models is defined as energy-based fracture failure after 
damage. In accordance with the fracture toughness $K_{\mathrm{IC}}$ of material, the critical strain energy release rate of the material can be obtained. The relationship between the critical strain energy release rate and the fracture toughness is as follows:

$$
G_{\mathrm{IC}}=\frac{K_{\mathrm{IC}}^{2}}{E^{\prime}}
$$

In Eq. (1), if it is a plane strain problem, $E^{\prime}=E / 1-v^{2}$. According to the research results in the existing literatures, the fracture toughness of osteon, interstitial bone and cement line can be obtained [12,17]. The material parameters of the osteon, interstitial bone, cement line and their critical strain energy release rates are shown in Table 1. A 4-node bilinear plane strain reduction integral element $\mathrm{CPE} 4 \mathrm{R}$ was used in the finite element analysis. The number of meshes for the twophase model and the three-phase model are 2557 and 2717 , respectively.

(a)

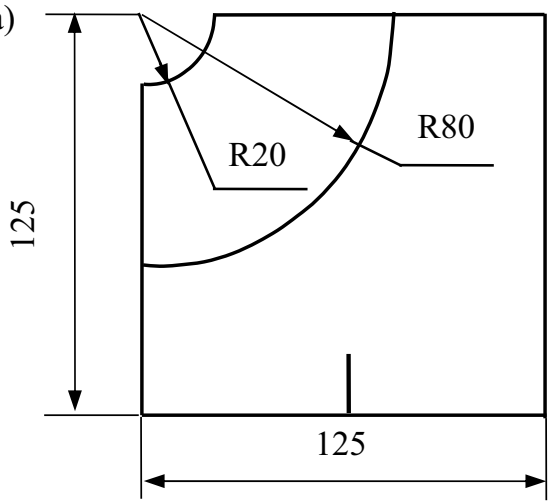

(b)

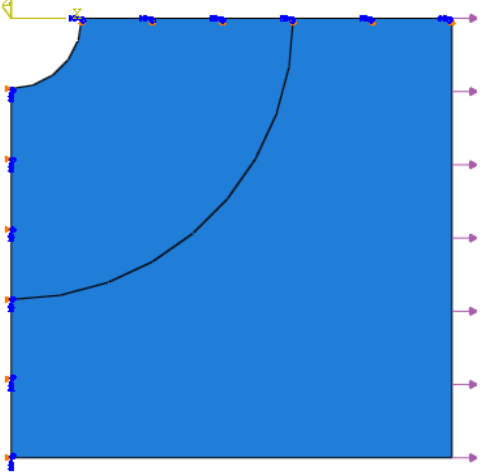

Fig. 2. Geometrical parameters (a) and boundary condition (b) of two-phase model (a)

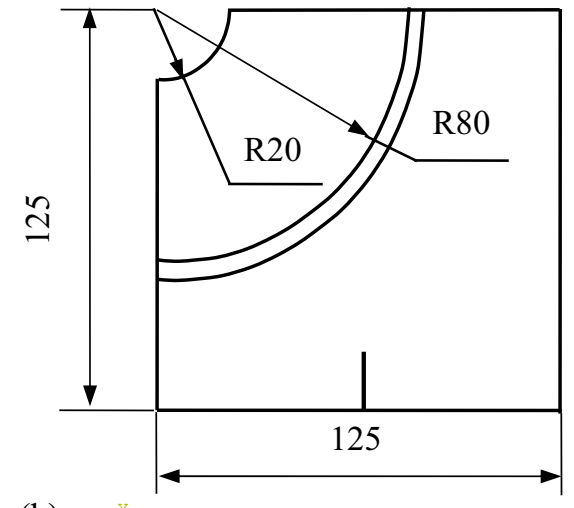

(b)

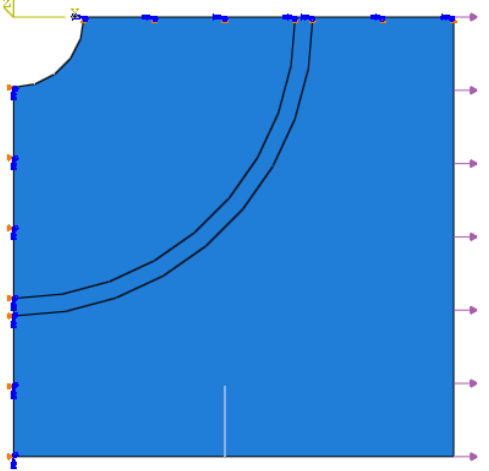

Fig. 3. Geometrical parameters (a) and boundary condition (b) of three-phase model

Table 1. Material parameter of different tissues of cortical bone ${ }^{[12 \cdot 17-18]}$

\begin{tabular}{|c|c|c|c|}
\hline Material & $\begin{array}{c}\text { Elasticity } \\
\text { modulus (GPa) }\end{array}$ & $\begin{array}{c}\text { Poisson's } \\
\text { ratio }\end{array}$ & $\begin{array}{c}\text { Strain energy } \\
\text { release rates } \\
(\mathrm{N} / \mathrm{mm})\end{array}$ \\
\hline One-phase & 10.46 & 0.167 & 0.422 \\
\hline Osteon & 9.13 & 0.17 & 0.86 \\
\hline $\begin{array}{c}\text { Interstitial } \\
\text { bone }\end{array}$ & 14.122 & 0.153 & 0.238 \\
\hline $\begin{array}{c}\text { Cement } \\
\text { line }\end{array}$ & 6.85 & 0.49 & 0.146 \\
\hline
\end{tabular}

\section{Results and Discussion}

The Mises stress distribution in the one-phase model and the two-phase model is approximately same when the cracks are initiated, but the stress distribution in the twophase and three-phase models is significantly different. The Mises stress distribution in the two-phase and threephase models are shown in Fig. 4. It can be seen from the Mises stress distribution that cement line significantly affects the stress distribution of the model. At the initiation of the crack, the displacement, initial strain, and applied stress at the reference point on the right side of the model are shown in Table 2. As it is shown in Table 2, the Mises stress of the three models is not much different at the crack initiation, but the crack initiation strains of the three models are significantly different. The initial strain of the two-phase model is 
$19.1 \%$ larger than that of the single-phase model, and the initial strain of the three-phase model is $57.8 \%$ larger than that of the two-phase model. The analysis results show that compared with the single-phase model, due to the microstructure of the osteon and the cement line in the two model, the initial strain required for crack initiation in the models are significantly increased due to the microstructure of the osteon and the cement line.

The above analysis results show that when the cortical bone is subjected to external loads, the osteons and cement lines of the cortial bone directly affect the stress and strain distribution of the model, and they can significantly increase the applied stress and strain for the initiation of cracks in cortical bone. This also demonstrates that osteons and cement lines are benefical to enhance the ability of cortical bone to resist fracture.

Table 2. Crack initiation condition of three different models

\begin{tabular}{|c|c|c|c|}
\hline Model & $\begin{array}{c}\text { Displacement } \\
(\mu \mathrm{m})\end{array}$ & $\begin{array}{c}\text { Initiation } \\
\text { strains }(\%)\end{array}$ & $\begin{array}{c}\text { Stress } \\
(\mathrm{MPa})\end{array}$ \\
\hline $\begin{array}{c}\text { One-phase } \\
\text { model }\end{array}$ & 0.04162 & 0.0373 & 2.2635 \\
\hline $\begin{array}{c}\text { Two-phase } \\
\text { model }\end{array}$ & 0.05791 & 0.0461 & 2.0813 \\
\hline $\begin{array}{c}\text { Three-phase } \\
\text { model }\end{array}$ & 0.1386 & 0.1092 & 1.7356 \\
\hline
\end{tabular}

When the external load $P$ is $35 \mathrm{MPa}$, the crack propagation path of the two models are shown in Fig. 5. It can be seen from Fig. 5 (a) that in the two-phase model, the crack directly penetrates the osteon boundary and enters the osteon during the crack propagation, and the propagation path is relatively straight. In the threephase model (Fig. 5b), the crack propagation path is deflected significantly away from the osteon, and when the crack tip approaches the osteon, the crack tip propagation towards the osteon. However, when the crack tip approaches the cement line, the crack propagation terminates, and when the applied stress is further increased, the microcrack will propagate and terminate along the interface between cement line and interstitial bone. It can be concluded that the cement line has the effect of preventing crack propagation, and can significantly change the path of microcrack propagation. The analysis results are consistent with the experimental observations of O'Brien et al. [19].
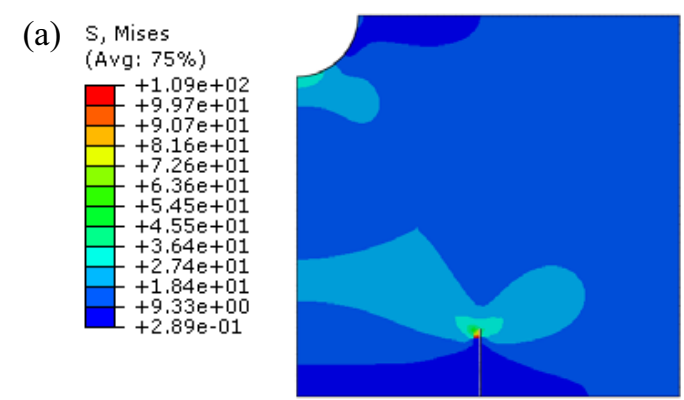

(b) $\mathrm{S}$, Mises
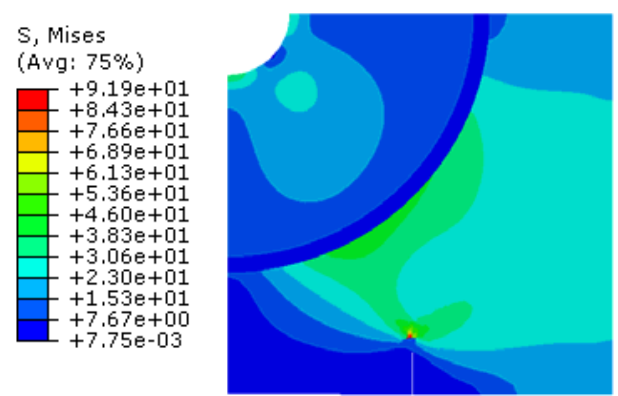

Fig 4. Stress distribution at crack initiation. (a) Two-phase model, (b) three-phase model

(a)
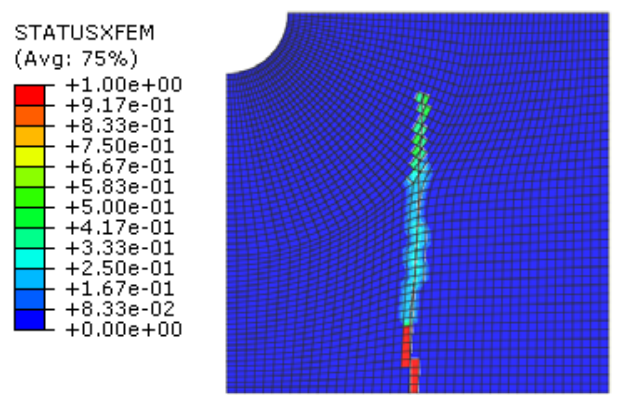

(b)

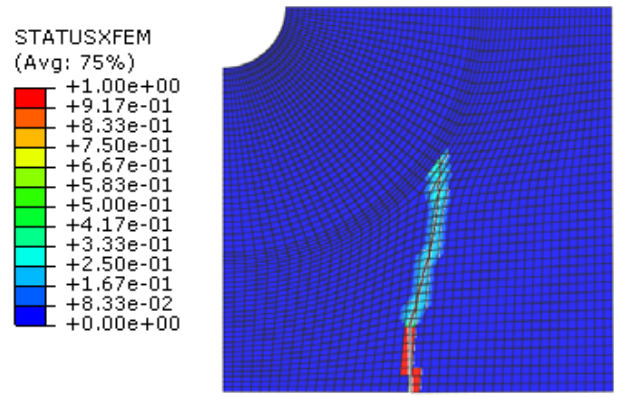

Fig. 5. Crack propagation paths of two-phase model (a) and three-phase model (b)

Comparing the analysis results of the crack propagation paths in the two models, it can be seen that under the same boundary conditions, the model with cement line can effectively change the micro crack propagation path and prevent the crack propagation. The deflection of the crack propagation path will dissipate more energy when the cortical bone is broken. Therefore, the cement lines in cortical bone can effectively increase the fracture resistance of bone and enhance the fracture toughness of bone. 


\section{Conclusions}

Based on the microstructure characteristics of cortical bone, three analysis models were established. The Abaqus X-FEM was used to analyze the stress and strain in the model, the microcrack propagation in the models were simulated, and the influence of the cement line and the osteon on the crack propagation path were compared and analyzed. the following conclusions can be drawn:

(1) According to the microstructure of the cortical bone, it can be seen that the cortical bone is composed of a cylindrical osteon and interstitial bone, and the osteon are unevenly distributed in the interstitial bone.

(2) The stress and strain distribution of the models are directly affected by osteon and cement line of the cortical bone, which can significantly increase the crack initiation strain of cortical bone, thereby improving the ability of cortical bone to resist fracture.

(3) The cement line can significantly change the propagation path of microcracks. The model with cement line can effectively change the propagation path of microcracks and deflect the crack propagation path. Thus, it will dissipate more energy when cortical bone fractures and enhance the fracture toughness of bone.

\section{Acknowledgments}

This work was supported by the Chongqing Research program of Basic Research and Frontier Technology (Grant No. cstc2018jcyjAX0805), and the Science and Technology Research Program of Chongqing Municipal Education Commission (Grant No. KJQN201903407).

\section{Reference}

1. S. Mischinski and A. Ural, Interaction of microstructure and microcrack growth in cortical bone: a finite element study. Comput. Method Biomec., 16(1), 81-94, 2013.

2. A. Gustafsson, N. Mathavan, M.J. Turunen, et al., Linking multiscale deformation to microstructure in cortical bone using in situ loading, digital image correlation and synchrotron X-ray scattering, Acta Biomaterialia, 69(15), 323-331, 2018.

3. D. Bajaj, J.R. Geissler, M.R. Allen, et al., The resistance of cortical bone tissue to failure under cyclic loading is reduced with alendronate. Bone, 64 (7): 57-64, 2014.

4. F.D. Liu, H. Ding, The changes of bone biomechanical properties in osteoporosis. J Med Biomech, 32(4): 388-392, 2017.

5. S.Manteghi, Z. Mahboob, Z. Fawaz, H. Bougherara, Investigation of the mechanical properties and failure modes of hybrid natural fiber composites for potential bone fracture fixation plates, J Mech. Behav. Biomed, 65, 306-316, 2016.

6. F.Libonati, L.Vergani, Understanding the structureproperty relationship in cortical bone to design a biomimetic composite. Composite Structures, 139(1): 188-198, 2016.
7. A. Demirtas, E. Curran, A. Ural, Assessment of the effect of reduced compositional heterogeneity on fracture resistance of human cortical bone using finite element modeling. Bone, 91, 92-101, 2016.

8. N.A. Raeisi, A.R. Arshi, M.R. Eslami, et al. Haversian cortical bone model with many radial microcracks:An elastic analytic solution. Med. Eng. Phys., 29(6): 708-717, 2007.

9. Y. Liu, B. Chen, G. Lu, X. Lin. Mechanical Experiment and Microscopic Analysis on Anisotropic Properties of Cortical Bone. J. of Medical Biomechanics, 33(5): 396-401, 2018.

10. A. Zheng, X. Luo, A mathematical programming approach for frictional contact problems with the extended finite element method, Archive of Applied Mechanics, 86, 599-616, 2016.

11. É. Budyn, T. Hoc, Multiple scale modeling for cortical bone fracture in tension using X-FEM[J]. European Journal of Computational Mechanics, 16(2): 213-236, 2007.

12. A.A. Abdel-Wahab, A.R. Maligno, V.V. Silberschmidt. Micro-scale modelling of bovine cortical bone fracture: Analysis of crack propagation and microstructure using X-FEM[J]. Computational Materials Science, 52(1): 128-135, 2012.

13. S. Li, A.A. Abdel-Wahab, V.V. Silberschmidt. Analysis of fracture processes in cortical bone tissue $[\mathrm{J}]$. Engineering Fracture Mechanics, 110: 448-458, 2013.

14. L. Hage, R. Hamade, Automatic Detection of Cortical Bones Haversian Osteonal Boundaries. AIMS Medical Science, 2(4): 328-346, 2015.

15. Cowin S C (Ed.). Bone mechanics handbook, 2nd Edition[M]. CRC Press, Boca Raton, 2001.

16. R.K. Nalla, J.H. Kinney, R.O. Ritchie, Mechanistic fracture criteria for the failure of human cortical bone. Nature materials, 2(3): 164-168, 2003.

17. L.P. Mullins, V. Sassi, P.E. McHugh, et al. Differences in the crack resistance of interstitial, osteonal and trabecular bone tissue. Annals of biomedical engineering, 37(12): 2574-2582, 2012.

18. É. Budyn, T. Hoc, Multiple scale modeling for cortical bone fracture in tension using X-FEM[J]. European Journal of Computational Mechanics, 16(2): 213-236, 2012.

19. F.J. O'Brien, D. Taylor, T.C. Lee, The effect of bone microstructure on the initiation and growth of microcracks. J. Orthop. Res., 23(2): 475-480, 2005. 